\title{
Aging studies for the CMS RPC system
}

\author{
Jan Eysermans ${ }^{* \dagger}$ \\ Facultad de Ciencias Físico-Matemáticas, Benemérita Universidad Autónoma de Puebla, \\ Mexico \\ E-mail: Gan.eysermansdcern.ch \\ María Isabel Pedraza Morales \\ Facultad de Ciencias Físico-Matemáticas, Benemérita Universidad Autónoma de Puebla, \\ Mexico \\ E-mail: maria.isabel.pedraza.moralesdcern.ch
}

Aging effects are studied for the Resistive Plate Chambers (RPC) at the Compact Muon Solenoid (CMS) experiment at the CERN Large Hadron Colider (LHC), which can manifest themselves during the High-Luminosity LHC (HL-LHC) running period. A dedicated consolidation program is set up using the CERN Gamma Irradiation Facility ++, where RPC detectors are exposed to a high gamma flux for a long term period equivalent to the HL-LHC operational time. Based on the past operational experience, the high background conditions are estimated and the RPC are tested under such circumstances. Several parameters are monitored as function of integrated charge and dedicated test beam periods allow measuring the detector efficiency as function of the background rate. In this work, an overview of the measurements which were performed for these studies is given. After having accumulated a significant amount of the total irradiation, no aging effects or degradation of the RPC detectors have been observed. These results suggest that the RPC system is capable to handle the HL-LHC conditions.

EPS-HEP 2017, European Physical Society conference on High Energy Physics

5-12 July 2017

Venice, Italy

\footnotetext{
${ }^{*}$ Speaker.

${ }^{\dagger}$ On behalf of the CMS muon group
} 


\section{Resistive Plate Chambers at CMS}

The Resitive Plate Chambers (RPC) at the Compact Muon Solenoid (CMS) experiment at the CERN Large Hadron Collider (LHC) provide redundancy to the muon trigger system and contributes to the muon reconstruction and identification[四][]. The CMS RPCs consist of 5 barrel stations having 480 chambers whereas the endcaps consist of 576 chambers distributed over 4 stations in both negative and positive sides.

A CMS RPC chamber consists of two gaps operated in avalanche mode to ensure reliable operation at high rates. Each gap consists of two $2 \mathrm{~mm}$ thick high resistive High-Pressure Laminates (HPL) separated by a $2 \mathrm{~mm}$ gas gap. A graphite coating at the outer surface of the HPL plates guaranties a uniform distribution of the charges to achieve a uniform electric field over the entire gap area. A non-flammable 3-component gas mixture of $95.2 \%$ freon $(\mathrm{C} 2 \mathrm{H} 2 \mathrm{~F} 4$, known as R134a), $4.5 \%$ isobutane (i-C4H10), and $0.3 \%$ sulphur hexafluoride (SF6) is used with a relative humidity of $40-50 \%$. The readout plane is located between both gaps and consists of strips aligned in the $\eta$ direction with a pitch between 2.28 and $4.10 \mathrm{~cm}$ in the barrel and between 1.74 and $3.63 \mathrm{~cm}$ in the encaps. In total, the entire system contains 137,000 of such copper strips covering an area of about $4000 \mathrm{~m}^{2}$. The strip signals are asynchronously sent to the Front End Boards (FEBs) which shapes the signal before being sent to the RPC linkboard system and the CMS data acquisition system. The FEBs are electronically controllable by means of the signal threshold to handle the noise of the detector.

\section{Motivation of longevity studies}

The increase of the luminosity during the High-Luminosity LHC (LH-LHC) phase up to $5 \times$ $10^{34} \mathrm{~cm}^{-2} \mathrm{~s}^{-1}$ at a center-of-mass energy of $\sqrt{s}=14 \mathrm{TeV}$ will be a challenge for the RPC system which can affect the muon identification and reconstruction in the following ways:

- Performance: increase of background rate which can lead to (partial) detector saturation and degradation of the performance;

- Aging: continuous irradiation can induce non-recoverable aging effects inside the RPC gap materials which can alter the material properties (e.g. bakelite resistivity).

Both effects can lead to a degradation of the performance with a lower muon detection efficiency. Therefore it is necessary to estimate the impact of these effects under the HL-LHC conditions up to an integrated luminosity of $3000 \mathrm{fb}^{-1}$.

Based on Run-I and II collision data, the maximum rates and integrated charge for the entire RPC system can be estimated, which is shown in the Table $\mathbb{U}$. A conservative safety factor of 3 is applied to cover all the uncertainties and to cope with e.g. the center of mass energy increase. During Run-I and II data taking, no detector degradation nor aging effects were observed so far.

\section{Methodology}

In order to provide answers to the above questions, a dedicated consolidation study was set up at the CERN Gamma Irradiation Facility $++(\mathrm{GIF}++)[$ [ $]$ ]. This facility consists of an intense 
Table 1: Rate and integrated charge for Run-I+II (up to 2016), expected HL-LHC conditions and expected HL-LHC conditions with safety factor[B]].

\begin{tabular}{lccc} 
& Run-I+II & HL-LHC & HL-LHC (+safety factor) \\
\hline Maximum rate $\left(\mathrm{Hz} / \mathrm{cm}^{2}\right)$ & $\approx 40$ & 200 & 600 \\
Max. Integrated charge $\left(\mathrm{mC} / \mathrm{cm}^{2}\right)$ & 4 & 280 & 840
\end{tabular}

$14 \mathrm{TBq}$ Cs-137 gamma source with controllable attenuation in order to obtain a wide range of gamma fluxes. Several weeks per year, dedicated muon test beam periods (providing intense 100 $\mathrm{GeV}$ muons) are available which allows to measure RPC efficiency subjected to different gamma background rates. Furthermore, the GIF ++ facility is a controlled environment with a constant temperature of about $21^{\circ} \mathrm{C}$, a constant humidity of $\approx 45 \%$ and gas monitoring system.

The setup consists of two endcap chambers of type RE2 and RE4 which are continuously irradiated (see Fig. W (left)). Also two non-irradiated chambers of the same type are installed to be used as reference. Periodic measurements are performed on both irradiated and reference chambers:

- continuous monitoring of the current and daily rate scans (irradiated chambers only);

- weekly noise scan and high voltage scan without radiation to monitor the Ohmic behavior;

- high voltage scans around the working point for different gamma fluxes;

- during test beam periods the chamber efficiency is measured;

- few times per year measurement of the bakelite resistivity.

From these measurements the detector performance and stability as function of integrated charge can be monitored.

\section{Preliminary results}

The longevity campaign has been started in July 2016 for the RE2 type chamber whereas the RE4 type has only been included in October 2016 due to gas limitations. The evolution of the

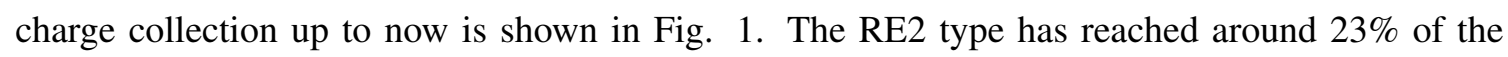
aimed charge collection whereas the RE4 type only has accumulated $8 \%$.

\section{Current stability}

The stability of the current as function of integrated charge is one of the key parameters to spot any aging effects. The current stability for both RE2 and RE4 is shown in Fig. $\square$ (left). In order to remove as much as possible the systematic fluctuations related to the gas system, atmospheric variations and external gamma attenuators, the current is normalized to the current of the corresponding reference chamber, taken at the same working point.

From the plot it is clear that a reasonable stable behavior is obtained up to the current values of the integrated charge for both RE2 and RE4. Small variations are accounted for different chamber conditions such as gas flow differences and overpressure. 

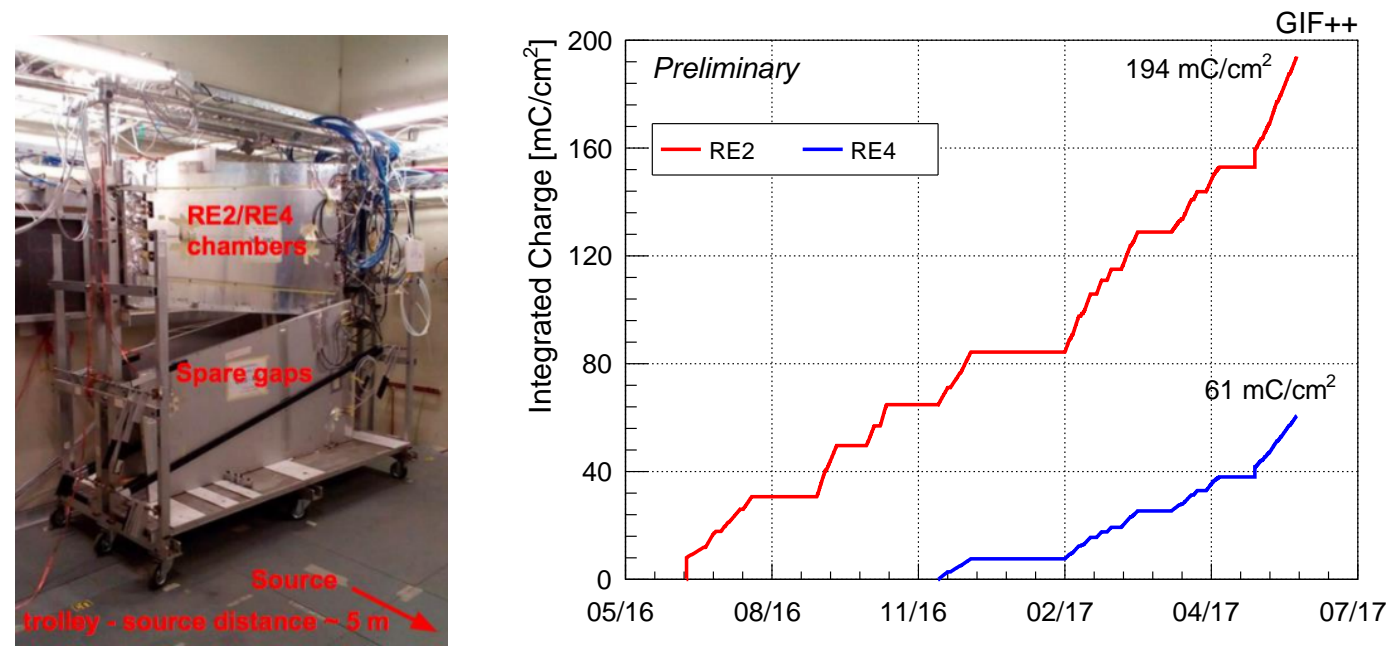

Figure 1: Left: GIF++ setup with the RE2/RE4 irradiated and reference chambers. Right: integrated charge accumulated at GIF++ for both RE2 and RE4 chambers.
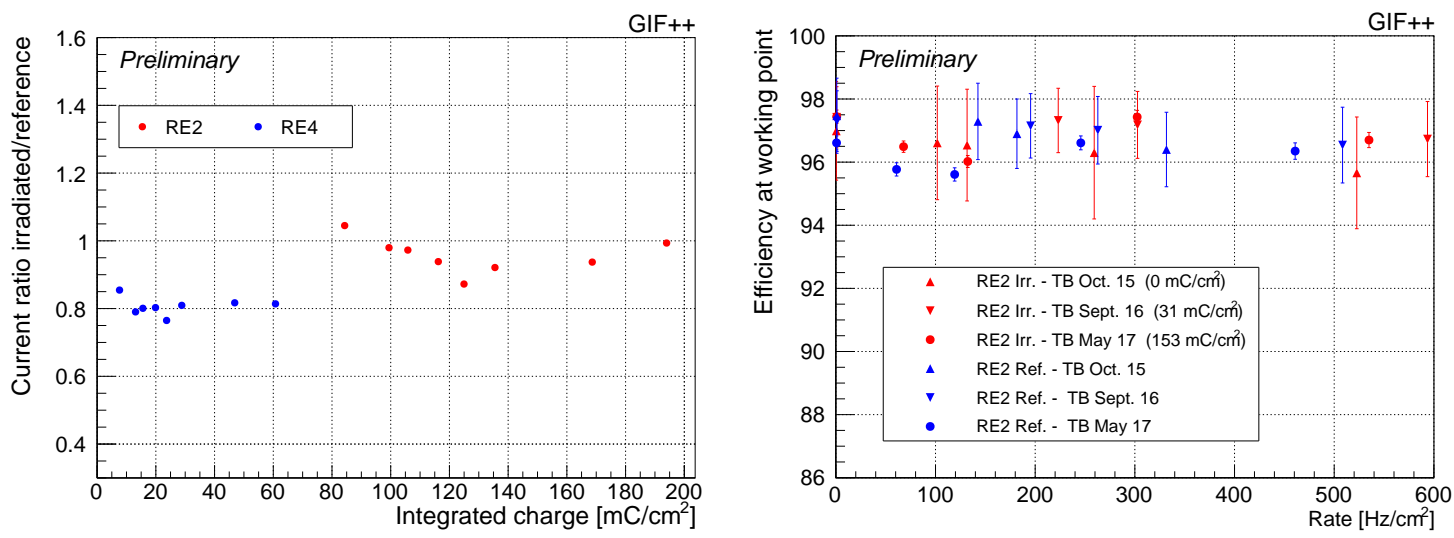

Figure 2: Left: current of the irradiated chamber divided by the rate and current of the reference chamber as function of the integrated charge of the irradiated chamber. Right: efficiency of the RE2 chamber at different values of integrated charge as function of the gamma background rate. The error bars correspond to different tracking algorithms used during the corresponding test beams.

\section{Efficiency and rate capability}

The final aim of the longevity studies is to show a high muon detection efficiency at sufficient high rates and stable efficiency up to the aimed charge collection. Dedicated test beam periods allows to measure precisely the muon efficiency for all the chambers under study.

In Fig. $\square$ (right), the efficiency is shown for both RE2 type chambers (irradiated and reference) at several values of integrated charge and different gamma background rates. No drop of efficiency is observed towards the maximum rate of $600 \mathrm{~Hz} / \mathrm{cm}^{2}$ nor up to an integrated charge of $153 \mathrm{mC} / \mathrm{cm}^{2}$. 


\section{Conclusions}

In this work the motivation, methodology and preliminary results of the CMS RPC aging campaign are presented. Continuous radiation of two RPC types under controlled conditions allows to monitor several detector parameters as function of integrated charge. After collecting of a significant amount of charge, no detector degradation or aging effects have been observed. The chambers tested with a mean background rate up to $600 \mathrm{~Hz} / \mathrm{cm}^{2}$ do not show any evidence of degradation in the detection performance. We conclude from the current study that the current RPC system is capable of reliable operation in the HL-LHC up to the given amounts of integrated charge.

\section{References}

[1] CMS Collaboration, "The CMS experiment at the CERN LHC", JINST 3 (2008) S0800.

[2] CMS Collaboration, "CMS, the Compact Muon Solenoid. Muon technical design report", CERN-LHCC-97-32

[3] Study of the CMS RPC detector performance in high radiation background conditions, Poster EPS-HEP2017

[4] CERN GIF++, "A new irradiation facility to test large-area particle detectors for the high-luminosity LHC program", M.R. Jaekel, M. Capeans, I. Efthymiopoulos, A. Fabich, R. Guida, G. Maire, M. Moll, D. Pfeiffer, F. Ravotti, H. Reithler; 2014, PoS(TIPP2014)102 